\title{
用等效线性系统模型法求伪码 自相关函数与功率谱
}

林可样陈揵

\section{一、引}

求伪码及其复码自相关函数的方法中, 㠇知的有概率法和解析计算法等 ${ }^{[1]}$. 一般来说，用 这些方法不能直接求出伪码及其复码的功率谱，而只能通过求自相关函数的宫氏变换来间接 地求出码的功率谱.

本文提出一种用等放线性系统模型求伪码及其复码自相关函数与功率谱的方法. 这种方 法的基本点是: 将伪码及其复码等效为一个由脉冲码激励的线性系统的输出, 从而使求码自 相关函数与功率谱的问题转化为求线性系统输出的自相关函数与功率谱的问题. 这样, 求码 自相关函数与功率谱便可各自分别进行.

等效线性系统模型方法的基本思想来自文献 [2]. 本文对此有所改进并加以完善化.

\section{二、数字序列、码序列、脉冲码波形与码浓形}

二进制数字序列 (简称数字序列) 作为空间的一个矢量表示为 $a=\left\{a_{n}\right\}$ ，度列元美属于 伽罗华二元域 $G F(2)$ ，只取 0 或 1 两个值，满足逻辑运算. 周期为 $p$ 的数字序列的自相关函 数定义为

$$
\rho_{a}(k)=\frac{A-D}{p},
$$

其中 $A$ 为 $a$ 序列与其 $k$ 循环移位后的 $a^{\prime}$ 序列对应元来一致的数目, 而 $D$ 为对应元素不一致的 数目.

二进制码序列 (简称码序列) 作为空间的矢量表示为 $b=\left\{b_{n}\right\}$, 码元取 1 或 -1 两个值, 满足普通算术运算. 码序列的自相关函数定义为

$$
\rho_{b}(k)=\frac{1}{p} \sum_{n=0}^{n-1} b_{n} b_{n+k} \text {. }
$$

二进制码波形(简称码波形)可由数字序列经两步变换而得. 第一步：分别用强度为 1 和 -1 的 8 函数代数字序列的元素 0 和 1 , 即

$$
\begin{aligned}
& 0 \longleftrightarrow \delta(t), \\
& 1 \longleftrightarrow-\delta(t) .
\end{aligned}
$$

于是, 数字序列 $a=\left\{a_{n}\right\}$ 变为二进制脉冲码序列（简称脉冲码序列) $a_{\delta}$, 


$$
a_{\delta}=\left\{b_{n} \delta(n)\right\}
$$

脉冲码序列 $a_{\delta}$ 的自相关函数定义为

$$
\rho_{a_{\delta}}(k)=\frac{A_{\delta}-D_{\delta}}{p}=\frac{1}{p} \sum_{n=0}^{p-1} b_{n} b_{n+k},
$$

其中 $A_{\delta}$ 和 $D_{\delta}$ 分别为 $a_{\delta}$ 与其 $k$ 循环移位序列 $a_{\delta}^{\prime}$ 中对应元素一致和不一致的数目. 当脉冲码 序列相邻元素之间为等间隔 $\Delta t$ 时, 便构成脉冲码波形

$$
a_{\delta}(t)=\sum_{n=0}^{p-1} b_{n} \delta(t-n \Delta t),
$$

$a_{8}(t)$ 的自相关函数为

$$
\begin{aligned}
\rho_{a \delta}(k \Delta t) & =\frac{1}{p \Delta t} \int_{-\Delta t / 2}^{p \Delta t-\Delta t / 2} d t \sum_{n=0}^{p-1} b_{n} b_{n+k} \delta(t-n \Delta t) \delta[t-(n+k) \Delta t] \\
& =\frac{1}{p \Delta t} \sum_{n=0}^{p-1} b_{n} b_{n+k} \delta(k \Delta t), k=0, \pm 1, \pm 2, \cdots .
\end{aligned}
$$

第二步：将脉冲码波形 $a_{\delta}(t)$ 通过一个脉冲响应函数为 $h_{0}(t)$ 的线性系统, 这里

$$
h_{0}(t)= \begin{cases}1, & 0 \leqslant t \leqslant \Delta t, \\ 0, & \text { 其它. }\end{cases}
$$

于是得到一个码波形

$$
a_{w}(t)=a_{\delta}(t) \otimes h_{0}(t)
$$

或写成

$$
a_{w}(t)=\sum_{n=0}^{p-1}\left[h_{0}(t) \otimes b_{n} \delta(t-n \Delta t)\right]=\sum_{n=0}^{p-1} b_{n} h_{0}(t-n \Delta t),
$$

上式中符号 $\otimes$ 表示卷积运算.

\section{三、等新线性香统搭型}

由于码波形可以等效为一个由相应脉冲码波形激励的, 具有脉冲响应 $h_{0}(t)$ 的线任系统 的输出,因此可以构造一个对应于码波形的等效线性系统模型,如图 1 所示.

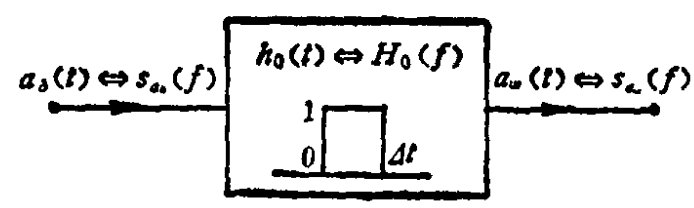

图 1

于是, 码波形 $a_{w}(t)$ 的频谱为

$$
S_{a_{w}}(f)=H_{0}(f) S_{a_{\delta}}(f),
$$

其中 $S_{a_{8}}(f)$ 为脉冲码波形 $a_{\delta}(t)$ 的频谱. 由此可得码波形 $a_{w}(t)$ 的功率谱为

$$
\phi_{a w}(f)=\phi_{0}(f) \phi_{a \delta}(f) \text {, }
$$

其中 $\phi_{a_{\delta}}(f)$ 为脉冲码波形 $a_{\delta}(t)$ 的功率谱, 而

$$
\phi_{0}(f)=\left|H_{0}(f)\right|^{2},
$$

可称为线性系统脉冲响应的能谱. 
对 (11) 式两边求富氏反变换,可得

$$
\rho_{a_{\nu}}(\tau)=\rho_{0}(\tau) \otimes \rho_{a_{\delta}}(\tau),
$$

其中 $\rho_{a_{w}}(\tau)$ 为码波形 $a_{\nu \nu}(t)$ 的自相关函数, $\rho_{a_{g}}(\tau)$ 为脉冲码波形 $a_{\delta}(t)$ 的自相关函数,而 $\rho_{0}(\tau)$ 可称为线珄系统脉冲响应 $h_{0}(t)$ 的自相关函数. 矩形脉冲响应 $h_{0}(t)$ 的自相关函数是一个峰值 为 $\Delta t$ 的三角形函数:

其中

$$
\rho_{0}(\tau)=\Delta t \Lambda_{\Delta t}(\tau),
$$

$$
\Lambda_{\Delta t}(\tau)=\left\{\begin{array}{l}
1-\frac{|\tau|}{\Delta t}, \quad|\tau| \leqslant \Delta t, \\
0, \text { 其它. }
\end{array}\right.
$$

$\rho_{0}(\tau)$ 的室氏变换 $\phi_{0}(f)$ 为

$$
\phi_{0}(f)-\Delta t^{2}\left(\frac{\sin \pi f \Delta t}{x f \Delta t}\right)^{2} .
$$

根据 (13) 式, 可以把码波形 $a_{\nu}(t)$ 的自相关函数 $\rho_{a_{w}}(\tau)$ 等效为一个由相应脉冲码波形自 相关函数 $\rho_{a_{\delta}}(\tau)$ 湤励的, 具有三角形脉冲响应 $\Delta t \Lambda_{\Delta t}(\tau)$ 的线性系统的输出, 这就是对应于码 波形自相关函数的等效线性系统模型,如图 2 所示.

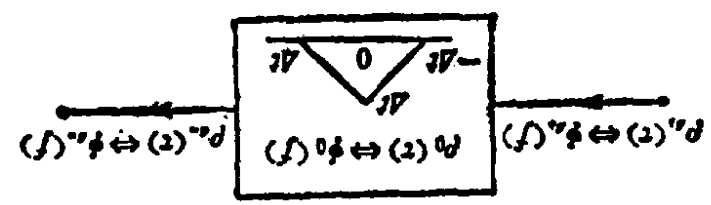

图 2

\section{四、伪码的自相关函数与功率谟}

根据等效线性系统模型, 可求出伪码波形的自相关函数与功率谱. 已经知道, 伪码序列 ( $m$ 序列)的自相关函数为

$$
\rho_{b}(k)=\left\{\begin{array}{l}
1, k-0, \pm p, \pm 2 p, \cdots, \\
-\frac{1}{p}, \text { 其它. }
\end{array}\right.
$$

于是, 根据 (6) 式, 可得相应的伪脉冲码波形 $a_{8}(t)$ 的自相关函数为

$$
\rho_{a_{g}}(k \Delta t)=\left\{\begin{array}{l}
\frac{1}{\Delta t} \delta(k \Delta t), \quad k=0, \pm p, \pm 2 p, \cdots, \\
-\frac{1}{p \Delta t} \delta(k \Delta t), \quad \text { 其它. }
\end{array}\right.
$$

(18) 式可分解为两个梳状函数之和, 因而可以改写成为:

$$
\rho_{a_{8}}(\tau)=\frac{p+1}{p \Delta t} \sum_{n=-\infty}^{\infty} \delta(\tau-n p \Delta t)-\frac{1}{p \Delta t} \sum_{n=-\infty}^{\infty} \delta(\tau-n \Delta t),
$$

对上式求富氏变换, 可得 $a_{\delta}(t)$ 的功率谱为:

$$
\phi_{a_{\delta}}(f)=\frac{p+1}{p^{2} \Delta t^{2}} \sum_{n=-\infty}^{\infty} \delta\left(f-\frac{n}{p \Delta t}\right)-\frac{1}{p \Delta t^{2}} \sum_{n=-\infty}^{\infty} \delta\left(f-\frac{n}{\Delta t}\right) .
$$


由 (13) 式及图 2 模型, 可得伪码波形 $a_{w}(t)$ 的自相关函数为:

$$
\rho_{a_{\omega}}(\tau)=\frac{p+1}{p} \sum_{n=-\infty}^{\infty} \Lambda_{\Delta t}(\tau-n p \Delta t)-\frac{1}{p} \cdot \sum_{n=-\infty}^{\infty} \Lambda_{\Delta t}(\tau-n \Delta t),
$$

其图形见图 3.
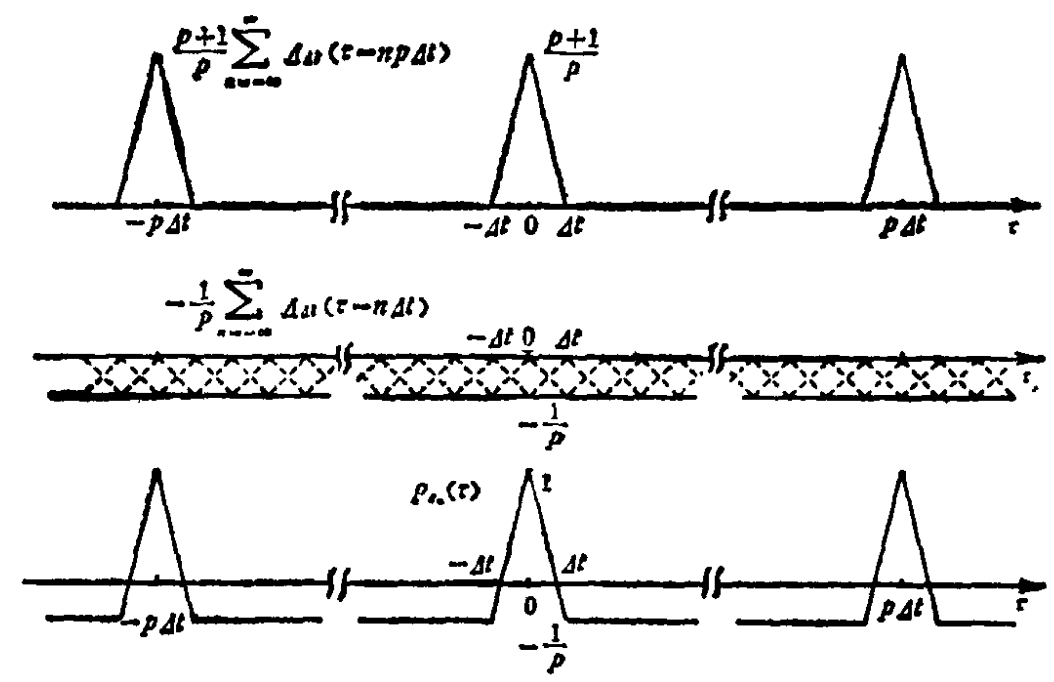

图 3

由图 3 可见,将伪脉冲码序列自相关函数离散值用直线段连接,所得到的图形就是相应的 伪码波形的自相关函数图形. 进一步推广，所有二进制码波形的自相关函数图形皆为其相应 二进制码序列的离散自相关函数值用直线段连接而成的图形.

根据 (13) 式,码波形的自相关函数等于其相应脉冲码序列的自相关函数与三角形脉冲响 应的卷积, 因此, 显然在离散点 $\tau=n \Delta t$ ( $n$ 为整数) 上 $\rho_{a_{u}}(\tau)$ 取值与 $\rho_{a_{\delta}}(\tau)$ 一致;而在相邻离 散值之间, $\rho_{a_{w}}(\tau)$ 是由两条不同斜率的直线段叠加而成. 不难证明, 由若干条不同斜率的直 线叠加，其结果仍然是一条直线. 于是， $\rho_{u s}(\tau)$ 在任意两个相邻离散点之间的图形是一条连 接相应离散点码序列自相关函数值的直线段。因此,只要求出码序列的自相关函数离散值,相 应的码波形自相关函数图形便随之确定了.

\section{五、复码的自相关函数与功率诸}

复码是由若干个子码按炤一定的逻辑函数关系组合而成的. 在一般情况下，图 1 和图 2 所示的等效线性系统模型对复码仍然适用，只不过这时的激励信号应改用相应的复脉冲码波 形及其自相关函数. 因此在应用等效线性系统模型时, 先要求出复脉冲码波形的自相关函数 (或功率谱),然后再求出图 2 所示的线性系统输出的自相关函数(或功率谱). 另外,在某些特 殊情况下，也可选择适当的等效线性系统脉冲响应和激励脉冲码波形的组合来求得复码波形 的自相关函数与功率谱. 在前一种情况下，问题集中到如何求复脉冲码波形的自相关函数与 功率谱上面. 这个问题拟另文专门讨论. 本文仅简单说明后一种情况. 为此, 下面讨论伪码 与二倍钟频码模 2 相加所组成的复码情况.

这时,复合数字序列 $x$ 为

$$
x=a \oplus 2 C l,
$$

其中 $a$ 为伪数字序列, $2 C l$ 为周期等于 $\Delta t$ 的钟频数字序列. 在这种情况下, 可构造一个如 
图 4 所示的线性系统模型,把复码波形 $x_{w}(2)$ 等效为这个系统的输出, 而系统的湤励信号仍为 $a$ 相应的脉冲码波形 $a_{\delta}(t)$.

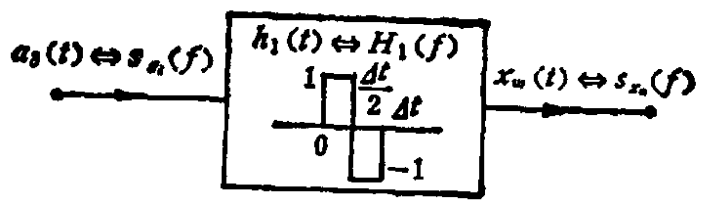

图 4

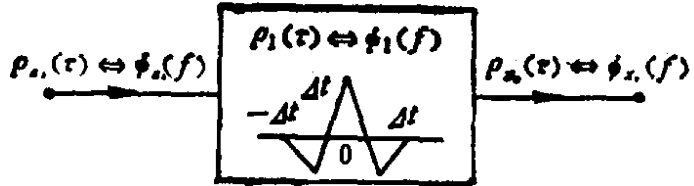

图 5

这个线性系统的输出、软入功率谱之间的关系为

其中

$$
\phi_{x_{w}}(f)=\phi_{1}(f) \phi_{a_{g}}(f),
$$

对 (23) 式两边求富氏反变换, 可得

$$
\phi_{1}(f)=\left|H_{1}(f)\right|^{2}
$$

$$
\rho_{x_{w}}(\tau)=\rho_{1}(\tau) \otimes \rho_{a_{\delta}}(\tau),
$$

其中 $\rho_{x_{w}}(\tau)$ 为复码波形的自相关函数,而 $\rho_{1}(\tau)$ 为 $h_{1}(t)$ 的自相关函数.

根据 (25) 式, 可把复码波形 $x_{w}(t)$ 的自相关函数等效为一个由伪脉冲码波形自相关函数 激励的、具有脉冲响应 $\rho_{1}(\tau)$ 的线性系统的输出, 如图 5 所示.

这里, 线性系统的脉冲响应为 $\rho_{1}(\tau)=\Delta t M_{1}(\tau)$, 其中

$$
M_{1}(\tau)=\left\{\begin{array}{l}
1-\frac{3}{\Delta t}|\tau|,|\tau| \leqslant \frac{\Delta t}{2}, \\
\frac{|\tau|}{\Delta t}-1, \quad \frac{\Delta t}{2} \leqslant|\tau| \leqslant \Delta t .
\end{array}\right.
$$

由 (19)、(25) 和 (26) 式, 可得

$$
\rho_{x_{w}}(\tau)=\frac{p+1}{p} \sum_{n=-\infty}^{\infty} M_{1}(\tau-n p \Delta t) \cdot \frac{1}{p} \sum_{n=-\infty}^{\infty} M_{1}(\tau-n \Delta t),
$$

$\rho_{x_{w}}(\tau)$ 的图形见图 6 .

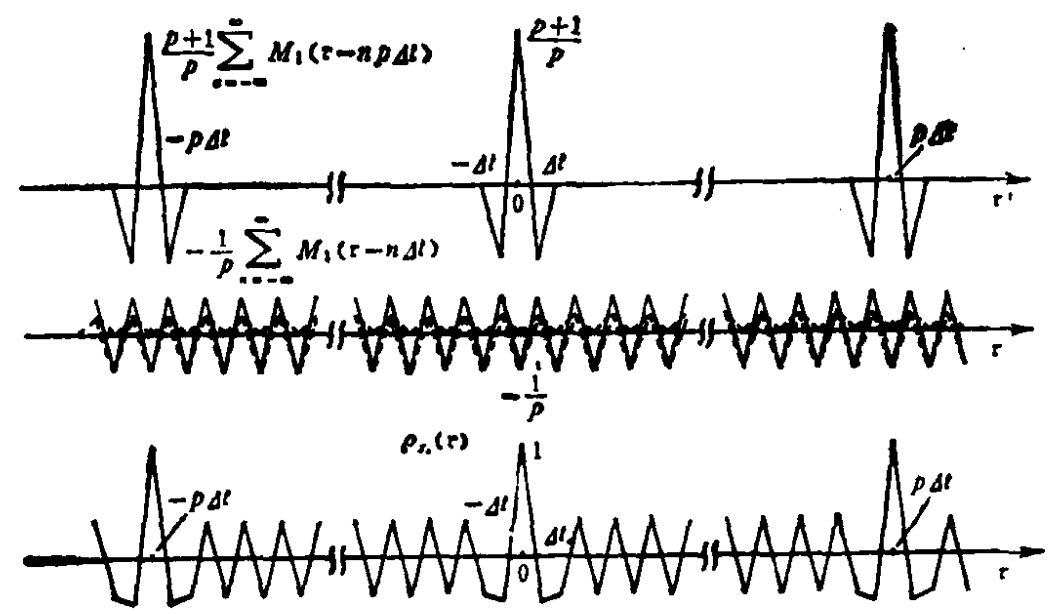

图 6

另外,由于图 4 所示线性系统的脉冲响应能谱为

$$
\phi_{1}(f)=\left|H_{1}(f)\right|^{2}=\Delta t^{2} \frac{\sin ^{4}(\pi f \Delta t / 2)}{(\pi f \Delta t / 2)^{2}},
$$


所以利用 (20)、(23) 和 (28) 式, 可得复码波形 $x_{w}(t)$ 的功率谱为

$$
\phi_{x_{w}}(f)=\frac{\sin ^{4}(\pi f \Delta t / 2)}{(\pi f \Delta t / 2)^{2}}\left[\frac{p+1}{p^{2}} \sum_{n=-\infty}^{\infty} \delta\left(f-\frac{n}{p \Delta t}\right)-\frac{1}{p} \sum_{n=-\infty}^{\infty} \delta\left(f-\frac{n}{\Delta t}\right)\right] .
$$

下面进一步求伪码与 $2 N^{\prime}$ 倍钟频码模 2 相加所组成的复码波形功率谱. 这时, 有

$$
x=a \oplus 2 \mathrm{~N}^{\prime} \mathrm{Cl}=a \oplus \mathrm{NCl},
$$

其中 $N=2 N^{\prime}$. 于是, 可构造复码波形的等效线性系统模型,如图 7 所示.

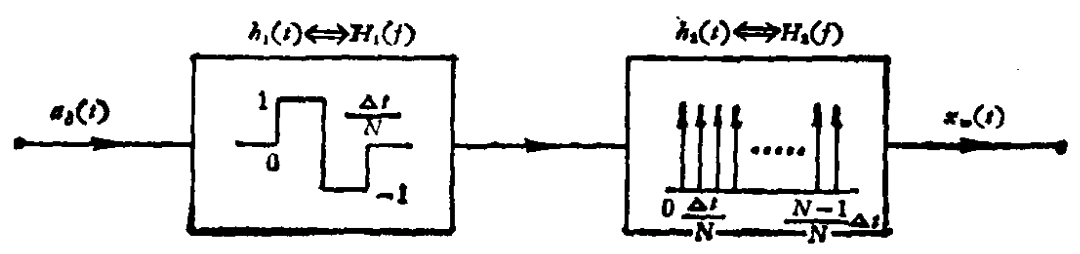

图 7

根据图 7, 可求得复码波形的功率谱为

$$
\phi_{x_{w}}(f)=\left|H_{1}(f)\right|^{2}\left|H_{2}(f)\right|^{2} \phi_{a_{\delta}}(f),
$$

其中

$$
\begin{aligned}
& \left|H_{1}(f)\right|^{2}=\frac{\Delta t^{2}}{N^{2}} \frac{\sin ^{4}\left(\frac{\pi f \Delta t}{2 N}\right)}{\left(\frac{\pi f \Delta t}{2 N}\right)^{2}}, \\
& \left|H_{2}(f)\right|^{2}=\left[\frac{\sin (\pi f \Delta t)}{\sin (\pi f \Delta t / N)}\right]^{2},
\end{aligned}
$$

于是可得

$$
\begin{aligned}
\phi_{x_{w}}(f)= & \frac{\sin ^{4}\left(\frac{\pi f \Delta t}{2 N}\right)}{N^{2}\left(\frac{\pi f \Delta t}{2 N}\right)^{2}}\left[\frac{\sin (\pi f \Delta t)}{\sin (\pi f \Delta t / N)}\right]^{2}\left[\frac{p+1}{p^{2}} \sum_{n=-\infty}^{\infty} \delta\left(f-\frac{n}{p \Delta t}\right)\right. \\
& \left.-\frac{1}{p} \sum_{n=-\infty}^{\infty} \delta\left(f-\frac{n}{\Delta t}\right)\right] .
\end{aligned}
$$

致谢: 本文承陈芳允和陈宗陆两位教授仔细审阅，特此致谢.

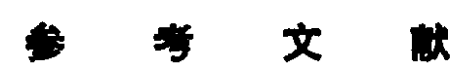

[1] 林可祥、汪一飞,伪随机码的原理与应用, 人民邮电出版社(将出版).

[2] James, D. Exact Power Spectrum of PN Codes, NASA CR-111002. 